\title{
BMJ IL-6 pathway-driven investigation Open of response to IL-6 receptor inhibition in rheumatoid arthritis
}

\author{
Jianmei Wang, ${ }^{1}$ Adam Platt, ${ }^{1}$ Ruchi Upmanyu, ${ }^{1}$ Søren Germer, ${ }^{2}$ Guiyuan Lei, ${ }^{1}$ \\ Christina Rabe, ${ }^{3}$ Ryma Benayed, ${ }^{2}$ Andrew Kenwright, ${ }^{1}$ Andrew Hemmings, ${ }^{4}$ \\ Mitchell Martin, ${ }^{2}$ Olivier Harari ${ }^{1}$
}

To cite: Wang J, Platt $A$, Upmanyu R, et al. IL-6 pathway-driven investigation of response to IL-6 receptor inhibition in rheumatoid arthritis. BMJ Open 2013;3: e003199. doi:10.1136/ bmjopen-2013-003199

- Prepublication history and additional material for this paper is available online. To view these files please visit the journal online (http://dx.doi.org/10.1136/ bmjopen-2013-003199).

JW and AP contributed equally.

Received 10 May 2013 Revised 16 July 2013 Accepted 24 July 2013

\footnotetext{
${ }^{1}$ Roche Products Ltd, Welwyn Garden City, UK ${ }^{2}$ Roche, Nutley, New Jersey, USA

${ }^{3}$ Roche Diagnostics GmbH, Penzberg, Germany

${ }^{4}$ Genentech (a member of the Roche group), South San Francisco, California, USA
}

Correspondence to Dr Olivier Harari; olivier.harari@roche.com

\section{ABSTRACT}

Objectives: To determine whether heterogeneity in interleukin-6 (IL-6), IL-6 receptor and other components of the IL-6 signalling pathway/network, at the gene, transcript and protein levels, correlate with disease activity in patients with rheumatoid arthritis (RA) and with clinical response to tocilizumab. Design: Biomarker samples and clinical data for five phase 3 trials of tocilizumab were analysed using serum (3751 samples), genotype (927 samples) and transcript (217 samples) analyses. Linear regression was then used to assess the association between these markers and either baseline disease activity or treatment response.

Results: Higher baseline serum IL-6 levels were significantly associated $(p<0.0001)$ with higher baseline DAS28, erythrocyte sedimentation rate, $C$ reactive protein and Health Assessment Questionnaire in patients whose responses to disease-modifying antirheumatic drugs (DMARD-IR) and to antitumour necrosis factor (aTNF-IR) were inadequate and patients who were naive/responders to methotrexate (MTX). Higher baseline serum IL-6 levels were also significantly associated with better clinical response to tocilizumab (versus placebo) measured by cDAS28 in the pooled DMARD-IR $(p<0.0001)$ and MTX-naive populations $(p=0.04)$. However, the association with treatment response was weak. A threefold difference in baseline IL-6 level corresponded to only a 0.17 -unit difference in DAS28 at week 16. IL-6 pathway single nucleotide polymorphisms and RNA levels also were not strongly associated with treatment response.

Conclusions: Our analyses illustrate that the biological activity of a disease-associated molecular pathway may impact the benefit of a therapy targeting that pathway. However, the variation in pathway activity, as measured in blood, may not be a strong predictor. These data suggest that the major contribution to variability in clinical responsiveness to therapeutics in RA remains unknown.

\section{INTRODUCTION}

Interleukin-6 (IL-6) is a pleiotropic cytokine important in autoimmune diseases, including

\section{ARTICLE SUMMARY}

\section{Article focus}

- This study aimed to determine whether genetic polymorphism and heterogeneity in expression of interleukin-6 (IL-6) pathway/network components could explain the variability in baseline disease activity and clinical response to tocilizumab in patients with rheumatoid arthritis (RA).

\section{Key messages}

- Higher baseline serum IL-6 levels were significantly associated with higher baseline disease activity (erythrocyte sedimentation rate, $\mathrm{C}$ reactive protein, Disease Activity Score using 28 joints (DAS28) and Health Assessment Questionnaire scores) in large populations of methotrexate-naive, disease-modifying antirheumatic drugs-inadequate responses (DMARD-IR) and antitumour necrosis factor-inadequate responses (aTNF-IR) patients with RA. Higher baseline serum IL-6 levels also showed significant correlation with better clinical response to tocilizumab (cDAS28) in DMARD-IR and methotrexate (MTX)-naive RA patients; however, the effect size was small, limiting the clinical usefulness of the marker in predicting treatment benefit. A threefold higher baseline IL- 6 concentration corresponded to only an additional 0.17 -unit decrease in DAS28 score in the DMARD-IR patients treated with tocilizumab.

- None of the genetic polymorphisms in IL-6 or IL-6R showed any association with treatment response to tocilizumab.

- No strong predictor of tocilizumab treatment response was identified; thus, the major factors underlying the patient-to-patient variability in clinical responsiveness to RA therapies remains unknown. This study illustrated well the difficulty of identifying clinically useful predictive biomarkers in a complex chronic disease.

rheumatoid arthritis (RA). ${ }^{1}$ High concentrations of IL- 6 are found in the synovial fluid and sera of patients with RA. IL-6 involvement in RA pathogenesis includes 


\section{ARTICLE SUMMARY}

Strengths and limitations of this study

- This study included well-characterised RA patients from five large phase 3 controlled clinical trials. Biomarker data were available for more than 3700 patients. The extensive collection of RNA, DNA and serum samples allowed thorough investigation of the target-related heterogeneity.

- This is the first demonstration of a significant association between baseline serum IL-6 levels and baseline disease activfindings of elevated serum IL-6 levels in RA patients compared with healthy controls.

- The current biomarker analysis is focused on clinical usefulness and included only blood samples. Any potentially useful information about local regulation of IL-6 pathway gene transcription and protein level in tissues involved in RA could not be obtained. ity in a large population of patients with RA, extending earlier

increased sIL-6R levels and RA susceptibility, ${ }^{11}{ }^{12}$ whereas a polymorphism at -174 in the promoter region of IL-6 (rs1800795) affects transcription activity and, thus, serum levels of IL- 6 . $^{13}$ Beyond RA, IL-6R polymorphisms that reduce cardiovascular risk ${ }^{14}{ }^{15}$ attenuate classical IL-6 signalling by increasing the cleavage rate of mIL-6R (rs2228145 and rs8192284) and mirror the effects of tocilizumab on levels of IL-6 (increase), C reactive protein (CRP) and fibrinogen (decrease). In asthma, the rs4129267 polymorphism in IL-6R was found to be associated with increased risk, and Ferreira et $a l^{16}$ suggested that an IL-6R antagonist may show efficacy in asthma in a genotype-dependent manner. The aim of this study was to determine whether baseline heterogeneity in IL-6, IL-6R and other components of the IL-6 signalling pathway/network, at the gene, transcript and protein levels, correlates with disease activity in RA and clinical response to tocilizumab.

\section{METHODS}

involvement in B-cell proliferation and cytokine production, matrix metalloproteinase expression, acute-phase response and anaemia. ${ }^{2}$

IL-6 signal transduction occurs through either a classical (cis-) or a trans-signalling pathway. In cis-signalling, IL-6 binds to membrane-bound IL-6 receptor $\alpha$ $(\mathrm{mIL}-6 \mathrm{R} \alpha)$ before forming a trimer with gp130 (encoded by IL-6 signal transducer (IL-6ST)). A signalling complex is formed when this heterotrimer forms a dimer with another IL-6/mIL-6R/gp130 complex. ${ }^{1}$ However, although gp130 is ubiquitously expressed, mIL-6R expression is restricted to hepatocytes and a subset of leucocytes. The trans-signalling pathway makes use of a soluble form of IL-6R (sIL-6R), to which IL-6 binds before forming a heterodimer with membranebound gp130, allowing cells that do not express mIL-6R to be activated by IL- $6 .{ }^{1}$

Studies of tocilizumab, ${ }^{3-8}$ a humanised monoclonal antibody targeted to IL-6R (membrane and soluble) that inhibits the interaction of IL-6 with its receptor, have shown meaningful amelioration of RA. However, the response is heterogeneous, consistent with the responses of other RA therapies. In a pooled analysis of patients who were inadequate responders to diseasemodifying antirheumatic drug (DMARD-IR) in pivotal trials, $39 \%$ of those receiving $8 \mathrm{mg} / \mathrm{kg}$ tocilizumab for 24 weeks failed to achieve American College of Rheumatology (ACR) 20 response (a 20\% improvement), whereas $18 \%$ did achieve ACR70 response (a $70 \%$ improvement). Therefore, there is great interest in discovering biomarkers to aid in physician decisionmaking by the accurate prediction of clinical response in individual patients.

IL-6 and IL-6R levels vary in the sera of RA patients. ${ }^{9} 10$ It has been suggested that some of this heterogeneity is genetically determined. The rs8192284 A/C polymorphism is found at the cleavage site of mIL-6R (Gln 357/Asp358) and has been associated with

\section{Patients and samples}

Serum specimens used in this study were collected from five pivotal phase 3 studies of tocilizumab in patients with RA: RADIATE, ${ }^{4}$ OPTION, ${ }^{3}$ TOWARD, ${ }^{5}$ AMBITION $^{6}$ and LITHE $^{7}$ (table 1). At baseline, serum samples were taken predose and at fasting, per protocol, resulting in the vast majority of them being collected in the morning. The distribution of the sampling times is shown in online supplementary figure S2. DNA samples were from RADIATE, OPTION and TOWARD, and RNA samples were from RADIATE. These trials evaluated tocilizumab ( 4 or $8 \mathrm{mg} / \mathrm{kg}$ ), administered every 4 weeks over a period of 24 weeks or longer, compared with placebo. The study populations differed according to background therapy with methotrexate (MTX) (OPTION, LITHE and RADIATE), any DMARD (TOWARD) or no DMARD (AMBITION). They also differed according to previous inadequate response to antitumour necrosis factor (aTNF) agents (RADIATE), MTX (OPTION, TOWARD and LITHE) or neither (AMBITION). In all studies, oral corticosteroids $(\leq 10 \mathrm{mg} /$ day prednisone or equivalent) were permitted if the dose was stable for at least 6 weeks before baseline. The study protocols were approved by relevant institutional review boards or ethics committees, and written informed consent was obtained from each patient. The studies were carried out in full concordance with the principles of the Declaration of Helsinki and with the laws and regulations of the country in which the research was conducted. DNA and RNA samples were collected only from patients who gave separate informed consent. DNA and RNA extraction methods are described in the online supplementary material.

\section{Serum analysis for IL-6 and sIL-6R}

Three assays for serum IL-6 levels were used to analyse samples from TOWARD, as follows: Quantikine Human 
Table 1 Demographics of patients from whom biological samples were collected and analysed and whose data passed a quality control check

\begin{tabular}{|c|c|c|c|c|c|c|c|c|c|}
\hline \multirow{2}{*}{$\begin{array}{l}\text { Sample type } \\
\text { Study }\end{array}$} & \multicolumn{3}{|l|}{ DNA } & \multirow{2}{*}{$\frac{\text { RNA }}{\text { RADIATE }}$} & \multicolumn{5}{|l|}{ Serum } \\
\hline & $\overline{\mathrm{OPTION}}$ & RADIATE & TOWARD & & $\overline{\mathrm{OPTION}}$ & LITHE & TOWARD & AMBITION & RADIATE \\
\hline \multirow[t]{2}{*}{ Patient population } & MTX-IR & aTNF-IR & DMARD-IR & aTNF-IR & MTX-IR & MTX-IR & DMARD-IR & MTX naive or free & aTNF-IR \\
\hline & 8 mg/kg & 8 mg/kg & 8 mg/kg & $8 \mathrm{mg} / \mathrm{kg}$ & $8 \mathrm{mg} / \mathrm{kg}$ & $8 \mathrm{mg} / \mathrm{kg}$ & $8 \mathrm{mg} / \mathrm{kg}$ & $8 \mathrm{mg} / \mathrm{kg}$ & $8 \mathrm{mg} / \mathrm{kg}$ \\
\hline \multirow[t]{4}{*}{ Treatment } & TCZ+MTX & TCZ+MTX & TCZ+DMARD & $\mathrm{TCZ}+\mathrm{MTX}$ & $\mathrm{TCZ}+\mathrm{MTX}$ & $\mathrm{TCZ}+\mathrm{MTX}$ & TCZ+DMARD & $\mathrm{TCZ}+\mathrm{MTX}$ & TCZ+MTX \\
\hline & 4 mg/kg & 4 mg/kg & & 4 mg/kg & $4 \mathrm{mg} / \mathrm{kg}$ & 4 mg/kg & & & 4 mg/kg \\
\hline & TCZ+MTX & TCZ+MTX & & $\mathrm{TCZ}+\mathrm{MTX}$ & $\mathrm{TCZ}+\mathrm{MTX}$ & TCZ+MTX & & & TCZ+MTX \\
\hline & MTX & MTX & DMARD & MTX & MTX & MTX & DMARD & MTX & MTX \\
\hline Total patients $(\mathrm{n})$ & 286 & 178 & 463 & 217 & 603 & 1126 & 1010 & 581 & 431 \\
\hline $\operatorname{TCZ}(\mathrm{n})$ & 189 & 119 & 301 & 151 & 409 & 753 & 661 & 244 & 289 \\
\hline $\operatorname{MTX}(n)$ & 97 & 59 & 162 & 66 & 194 & 373 & 349 & 337 & 142 \\
\hline Age (years) & $51.3(11.1)$ & $51.5(12.4)$ & $54.2(12.6)$ & 53.2 (11.9) & $51.0(12.2)$ & $51.9(12.4)$ & $53.4(12.9)$ & $50.3(13.0)$ & $52.9(12.6)$ \\
\hline Female (\%) & 79.0 & 79.8 & 81.0 & 82.40 & 81.8 & 82.9 & 81.7 & 80.4 & 81.4 \\
\hline Height (cm) & $163(9)$ & $165(8)$ & $164(9)$ & $165(8)$ & $162(9)$ & $162(9)$ & $163(9)$ & $163(9)$ & $165(8)$ \\
\hline Weight (kg) & $73(17)$ & 77 (19) & $76(18)$ & $77(20)$ & 79 (17) & $73(19)$ & $74(19)$ & $73(18)$ & $75(18)$ \\
\hline RA duration (years) & $7.8(7.3)$ & $11.1(8.3)$ & $9.7(9.1)$ & $12.1(9.6)$ & $7.6(7.3)$ & $9.2(8.0)$ & $9.6(8.8)$ & $6.4(8.2)$ & $11.6(9.1)$ \\
\hline RF positive (\%) & 76.2 & 79.8 & 75.6 & 76.5 & 77.6 & 82.1 & 77.1 & 72.5 & 75.2 \\
\hline White (\%) & 73.8 & 93.3 & 76.2 & Not applicable & Not applicable & Not applicable & Not applicable & Not applicable & Not applicable \\
\hline TCZ (n) & 141 & 109 & 233 & Not applicable & Not applicable & Not applicable & Not applicable & Not applicable & Not applicable \\
\hline $\operatorname{MTX}(n)$ & 70 & 57 & 120 & Not applicable & Not applicable & Not applicable & Not applicable & Not applicable & Not applicable \\
\hline DAS28 & $6.8(0.9)$ & $6.8(1)$ & $6.6(1)$ & $6.8(0.9)$ & $6.8(0.9)$ & $6.5(0.9)$ & $6.7(1.0)$ & $6.8(0.9)$ & $6.8(1.0)$ \\
\hline $\mathrm{HAQ}$ & $1.6(0.6)$ & $1.7(0.6)$ & $1.5(0.6)$ & $1.7(0.6)$ & $1.6(0.6)$ & $1.5(0.6)$ & $1.5(0.6)$ & $1.5(0.6)$ & $1.7(0.6)$ \\
\hline SJC & 20.9 (11.3) & $18.8(10.6)$ & 20.5 (11.9) & $18.1(9.8)$ & $20.0(11.2)$ & $16.9(9.4)$ & $19.6(11.5)$ & $19.8(11.2)$ & $18.9(10.6)$ \\
\hline TJC & $32.3(15.2)$ & $31.2(15.2)$ & $30.1(15.8)$ & $31.4(15.1)$ & 32.6 (15.6) & $28.3(14.7)$ & $30.0(15.7)$ & $31.9(14.4)$ & $31.1(15.6)$ \\
\hline CRP & $2.4(2.9)$ & $3.7(4.2)$ & $2.3(2.9)$ & $3.3(3.8)$ & $2.6(3.0)$ & $2.2(2.5)$ & $2.6(3.8)$ & $3.0(3.3)$ & $3.2(3.8)$ \\
\hline
\end{tabular}

Values with numbers in parentheses are mean (SD).

aTNF, antitumour necrosis factor; CRP, C reactive protein; DAS28, Disease Activity Score using 28 joints; DMARD, disease-modifying antirheumatic drug; HAQ, Health Assessment Questionnaire; IR, inadequate responder; MTX, methotrexate; RA, rheumatoid arthritis; RF, rheumatoid factor; SJC, swollen joint count; TCZ, tocilizumab; TJC, tender joint count. 
IL-6 Immunoassay (R\&D Systems Inc, Minneapolis, Minnesota, USA), performed by Huntington Life Sciences (Huntington, UK) according to the instructions of the manufacturer (Roche Diagnostics, Penzberg, Germany) of the IL- 6 assay ${ }^{17}$; IMPACT platform, ${ }^{18}$ performed by Roche Diagnostics; and RBM IL-6 assay, performed by Myriad RBM (Austin, Texas, USA). Given the tight correlations among these three assays (see online supplementary figure S1A), we chose to analyse data from the Quantikine Human IL-6 Immunoassay (R\&D Systems Inc) because they were available for nearly all patients in all five core studies. The limit of detection of this IL-6 assay is $3.12 \mathrm{pg} / \mathrm{mL}$. The assay is unaffected by the addition of recombinant human sIL-6 $\mathrm{R}^{19}$; therefore, it can be assumed that the data reflect the total concentration of IL-6 (ie, the concentration of free IL-6 plus the amount of IL-6 bound to sIL-6R).

Serum concentrations of endogenous sIL-6R were measured by Huntington Life Sciences using the Quantikine Human sIL-6R Immunoassay (R\&D Systems Inc) according to the manufacturer's instructions. The sensitivity of the sIL-6R assay is $31.2 \mathrm{pg} / \mathrm{mL}$. Measured sIL-6R concentrations are insensitive to the addition of recombinant human IL- 6 but are sensitive to the presence of tocilizumab. ${ }^{20}$ Therefore, it can be assumed that the sIL-6R data presented reflect the total amount of free sIL-6R plus sIL-6R complexed with IL-6 plus (in samples that contain tocilizumab), an unquantified fraction of sIL-6R bound by tocilizumab.

\section{Genotyping}

DNA samples were genotyped using HumanHap550k BeadChip V 3.0 (Illumina, San Diego, California, USA) and custom chips using the OPA genotyping assay. Quality control (QC) methods for DNA samples and genotyping data are described in the online supplementary material. High-quality markers with call rates $\geq 95 \%$ were included in the analysis. To reduce the multiplicity of hypothesis testing, a targeted approach was adopted. Three groups of genes were considered in this analysis. Tier 1 markers consisted of 26 polymorphisms within the $I L-6$ and $I L-6 R$ regions (see online supplementary table S1). Ten single nucleotide polymorphisms (SNPs) were selected in the IL-6 region, which is defined as $5 \mathrm{~kb}$ upstream and $5 \mathrm{~kb}$ downstream of the transcription coordinate; the SNPs cover a region of $12.6 \mathrm{~kb}$. Similarly, 18 SNPs were selected in the IL-6R region, covering a region of $62.2 \mathrm{~kb}$. The association of these markers with treatment benefit represents primary hypotheses. Tier 2 markers consisted of 233 polymorphisms within 22 genes identified as in the IL-6 pathway, termed the IL-6 canonical pathway (see online supplementary material). Tier 3 markers consisted of 1005 polymorphisms in 67 genes, identified with GeneGo (Carlsbad, California, USA) software, related to IL-6 signalling, herein termed the IL-6 network (see online supplementary material). Markers for tiers 2 and 3 correspond to the decreasing strength of the hypothesis and the increasing dimensions compared with tier 1 markers. This approach provides greater power in the confirmatory analysis for the primary biomarker hypothesis, yet allows for focused exploratory analysis to address the secondary hypotheses.

PCR-based HLA-DRB1 typing was performed on the DNA samples independently of the genotyping using whole genome and custom arrays. The number of shared epitope alleles $(0,1$ or 2$)$ was determined for each patient based on his or her HLA-DRB1 genotype.

\section{Transcript analysis}

Two hundred and thirty-three RNA samples, prepared from whole blood, at baseline were analysed using the GeneChip Human Genome U133 Plus 2.0 Array (Affymetrix, Santa Clara, California, USA). After samples that failed quality control tests were removed (see online supplementary material), data for the remaining 217 samples were normalised using the Robust MultiArray Analysis normalisation $^{21}$ (Bioconductor). Transcripts for $I L-6, I L-6 R$ and $I L-6 S T$ were extracted from the normalised data. IL-6 transcript levels were not analysed because of the low levels of signal observed. Three probe sets targeting $I L-6 R$ were analysed. Of those, 205945_at and 217489_at expression levels were found to be strongly correlated (Pearson's $r^{2}=0.75$ ), and the third probe set, 226333_at, had a weaker correlation with the other two (Pearson's $r^{2}=0.34$ and 0.38 ). These three probe sets represent the primary hypothesis in RNA transcripts. In addition, seven probe sets from IL-6ST were analysed as secondary markers.

\section{Measures of disease activity and clinical response}

Six different measures were used for disease activity at baseline: erythrocyte sedimentation rate (ESR), CRP, swollen joint count at 28 joints (SJC28), tender joint count at 28 joints (TJC28) and Health Assessment Questionnaire (HAQ), with disease activity at 28 joints (DAS28; derived from the four core components of ESR, SJC, TJC and patient global assessment) considered the primary end point. As a measurement of treatment response, change in DAS28 from baseline at week 16 (cDAS28) was mainly used. Unlike dichotomous responder criteria (eg, ACR response, DAS28 remission and clinical disease activity index remission), this continuous measure captures the range of individual responses and is, therefore, more sensitive in detecting the effect of biomarkers across different levels of prevalence. In all studies except AMBITION, patients were allowed to enter escape therapy at 16 weeks if they had inadequate responses. Therefore, cDAS28 was used at week 16 to increase the sample size and to minimise bias.

\section{Statistical analysis}

Table 2 provides a summary of all markers analysed. Quality control analyses were performed for genotyping and gene expression data, as described in the online supplementary material; assays and samples with poor 
Table 2 Summary of markers investigated

\begin{tabular}{|c|c|c|c|}
\hline $\begin{array}{l}\text { Sample } \\
\text { type }\end{array}$ & Target gene & Analytes (n) & Assay (manufacturer) \\
\hline \multicolumn{4}{|l|}{ Primary } \\
\hline \multirow[t]{2}{*}{ DNA } & $I L-6$ & 10 SNPs & Bead-Chip arrays HumanHap550k V.3.0, OPA custom array \\
\hline & IL-6R & 18 SNPs & (Illumina) \\
\hline RNA & IL-6R & 3 probe sets & GeneChip Human Genome U133 Plus 2.0 Array (Affymetrix) \\
\hline \multirow{2}{*}{ Serum } & IL-6 & 1 & Human IL-6 Immunoassay (Quantikine) \\
\hline & sIL-6R & 1 & Human sIL-6R Immunoassay (Quantikine) \\
\hline \multicolumn{4}{|c|}{ Secondary } \\
\hline \multirow[t]{2}{*}{ DNA } & $\begin{array}{l}\text { IL-6 network } \\
\text { genes }\end{array}$ & 233 SNPs (22 genes) & $\begin{array}{l}\text { Bead-Chip arrays HumanHap550k V.3.0, OPA custom array } \\
\text { (Illumina) }\end{array}$ \\
\hline & $\begin{array}{l}\text { IL-6 pathway } \\
\text { genes }\end{array}$ & $\begin{array}{l}1004 \text { SNPs }(67 \\
\text { genes) }\end{array}$ & \\
\hline RNA & IL-6ST & 7 probe sets & GeneChip Human Genome U133 Plus 2.0 Array (Affymetrix) \\
\hline
\end{tabular}

data quality were removed. Distribution was assessed for each marker, and appropriate transformation was applied before further analysis. Minor allele frequencies and Hardy-Weinberg equilibrium were calculated for SNPs. Given that the distribution within each gene expression probe set was approximately normal, no further transformation was applied. Log transformation was applied to the protein markers IL-6 and sIL-6R because of the skewed distribution of raw values.

Correlations among the baseline markers were assessed within each sample type for an understanding of the amount of information shared among the assays. For SNPs, linkage disequilibrium coefficients within IL-6 and IL-6R were obtained from HapMap 22 based on the CEU (Utah residents of Northern and Western European ancestry from the CEPH collection) data. For the gene expression markers, correlation coefficients were calculated among probe sets within $I L-6 R$ and $I L-6 S T$. For the proteins, correlation was calculated among the three assays for IL-6 and between IL-6 and sIL-6R. Pearson or Spearman rank correlation was used as appropriate.

Linear regression was used to assess the association between markers with baseline disease activity and markers with treatment response. Because of the skewed distribution of the raw values, $\log (\mathrm{ESR})$ and $\log (\mathrm{CRP}+1)$ were used in the regression analysis. All patients were included in the regression analysis of baseline disease activity. In the regression analysis of cDAS28, separate analyses were undertaken in patients treated with tocilizumab and those administered placebo. Given that baseline DAS28 is strongly associated with change in DAS28 at week 16, it was included as a covariate in all models. No additional covariates were included in the model. In addition, the number of shared epitope alleles ${ }^{22}(0,1$ or 2) and the tocilizumab dose were used as covariates in the analysis of genetic data.

The numbers of genetic markers tested were 18233 and 1004, respectively, for the tiers 1, 2 and 3 markers.
In addition, six baseline end points were used on the baseline disease association analysis. Analyses were undertaken in the white population and the all-patient population. To adjust for multiple testing, the false discovery rate $(\text { FDR })^{23}$ was calculated within each tier and within the white population and the all-patient population. This FDR accounted for the multiplicity of markers and the end points.

To help interpret the outcome of the regression analysis and to enable direct comparison of the analysis results across all markers, consistent representations of the results were used. For continuous markers, the regression coefficients were standardised to reflect how many units of change in the end points corresponded to a difference of $1 \mathrm{SD}$ in the marker value. For the SNP markers, an allelic model was used (ie, two copies of the minor allele had twice the effect of one copy). The regression coefficient corresponded to the change in end point per copy of minor allele.

\section{RESULTS}

\section{Baseline characteristics of patients with DNA, RNA and} serum samples

Baseline characteristics of the patient subgroups with DNA, RNA and serum samples available are shown in table 1 . They are generally comparable to the overall population for each study. Patient ethnicity is relevant to the genetic analysis. Therefore, the proportion of patients of European ancestry (White) is shown for the DNA subpopulations. Of the 927 patients with DNA samples, $730(79 \%)$ were of European ancestry (White; table 1). As expected, based on treatment experience, patients from the MTX-naive or the MTX-free study had shorter disease duration and those from the aTNF-IR study had longer disease duration. For serum markers where large sample size was available, data were analysed separately for the MTX-naive/MTX-free population, 
Table 3 Association of markers with baseline disease activity

\begin{tabular}{|c|c|c|c|c|c|c|c|c|}
\hline \multicolumn{9}{|c|}{ Serum protein and RNA markers } \\
\hline \multirow[b]{2}{*}{$\begin{array}{l}\text { End } \\
\text { point }\end{array}$} & \multicolumn{3}{|l|}{ Serum IL-6 } & \multicolumn{3}{|c|}{ Serum sIL-6R } & \multirow{2}{*}{$\begin{array}{l}\text { IL-6R } \\
\text { 217489_s_at } \\
\text { RADIATE }\end{array}$} & \multirow{2}{*}{$\begin{array}{l}\text { IL-6ST } \\
\text { 234967_a } \\
\text { RADIATE }\end{array}$} \\
\hline & AMBITION & RADIATE & $\begin{array}{l}\text { Pooled } \\
\text { DMARD-IR }\end{array}$ & AMBITION & RADIATE & $\begin{array}{l}\text { Pooled } \\
\text { DMARD-IR }\end{array}$ & & \\
\hline \multicolumn{9}{|c|}{ DAS28 } \\
\hline $\mathrm{N}$ & 480 & 322 & 2286 & 577 & 423 & 2620 & 217 & 217 \\
\hline$\beta$ & 0.209 & 0.257 & 0.201 & -0.007 & 0.075 & 0.054 & 0.109 & -0.04 \\
\hline$r^{2}$ & 0.051 & 0.072 & 0.044 & 0 & 0.006 & 0.003 & 0.013 & 0.002 \\
\hline$p$ & $<0.0001$ & $<0.0001$ & $<0.0001$ & 0.8478 & 0.1018 & 0.0044 & 0.09 & 0.54 \\
\hline \multicolumn{9}{|l|}{ TJC } \\
\hline $\mathrm{N}$ & 481 & 327 & 2305 & 580 & 428 & 2642 & 217 & 217 \\
\hline$\beta$ & -0.186 & 1.455 & 0.138 & -0.154 & 1.28 & 0.054 & 0.27 & 1.276 \\
\hline$r^{2}$ & 0 & 0.009 & 0 & 0 & 0.007 & 0 & 0 & 0.007 \\
\hline $\mathrm{p}$ & 0.7742 & 0.0938 & 0.6611 & 0.7967 & 0.0889 & 0.8558 & 0.79 & 0.2140 \\
\hline \multicolumn{9}{|l|}{ SJC } \\
\hline $\mathrm{N}$ & 481 & 327 & 2305 & 580 & 428 & 2642 & 217 & 217 \\
\hline$\beta$ & 0.247 & 1.421 & 0.813 & -0.679 & -0.069 & 0.52 & -0.864 & 0.218 \\
\hline$r^{2}$ & 0 & 0.018 & 0.006 & 0.004 & 0 & 0.002 & 0.008 & 0 \\
\hline $\mathrm{p}$ & 0.6279 & 0.0158 & 0.0003 & 0.1424 & 0.8938 & 0.013 & 0.1950 & 0.7440 \\
\hline \multicolumn{9}{|l|}{ ESR } \\
\hline $\mathrm{N}$ & 481 & 327 & 2299 & 580 & 428 & 2635 & 217 & 217 \\
\hline$\beta$ & 0.19 & 0.172 & 0.151 & 0.039 & -0.003 & 0.023 & 0.164 & -0.132 \\
\hline$r^{2}$ & 0.088 & 0.091 & 0.055 & 0.004 & 0 & 0.001 & 0.082 & 0.053 \\
\hline $\mathrm{p}$ & $<0.0001$ & $<0.0001$ & $<0.0001$ & 0.132 & 0.9158 & 0.0672 & $<0.0001$ & 0.0007 \\
\hline \multicolumn{9}{|l|}{ CRP } \\
\hline $\mathrm{N}$ & 481 & 327 & 2305 & 580 & 428 & 2642 & 217 & 217 \\
\hline$\beta$ & 0.363 & 0.429 & 0.35 & 0.007 & 0.019 & 0.019 & 0.228 & -0.145 \\
\hline$r^{2}$ & 0.288 & 0.358 & 0.309 & 0 & 0.001 & 0.001 & 0.089 & 0.036 \\
\hline \multirow{2}{*}{\multicolumn{9}{|c|}{$\mathrm{HAQ}$}} \\
\hline & & & & & & & & \\
\hline $\mathrm{N}$ & 480 & 324 & 2165 & 579 & 425 & 2465 & 217 & 217 \\
\hline$\beta$ & 0.085 & 0.145 & 0.084 & 0.002 & 0.074 & 0.024 & 0.048 & -0.103 \\
\hline$r^{2}$ & 0.019 & 0.062 & 0.018 & 0 & 0.016 & 0.002 & 0.007 & 0.031 \\
\hline$p$ & 0.0022 & $<0.0001$ & $<0.0001$ & 0.933 & 0.0089 & 0.0504 & 0.22 & 0.009 \\
\hline
\end{tabular}

DNA markers

\begin{tabular}{llllllllllll} 
Tiers & Gene & Chr & SNP & Population & MAF & End point & $\mathbf{n}$ & $\boldsymbol{\beta}$ & SE & Raw P & FDR P \\
\hline 1 & IL-6R & 1 & rs1386821 & White & 0.17 & HAQ & 708 & 0.135 & 0.041 & 0.001 & 0.18 \\
& & & & All & 0.15 & HAQ & 845 & 0.118 & 0.040 & 0.004 & 0.52 \\
3 & TOLLIP & 11 & rs5743899 & All & 0.22 & ESR & 893 & 0.15 & 0.03449 & $1.5 \mathrm{E}-05$ & 0.046 \\
& RUNX1 & 21 & rs2252585 & All & 0.32 & ESR & 905 & 0.1209 & 0.02884 & $3.0 \mathrm{E}-05$ & 0.046 \\
& IL-1RAPL1 & 23 & rs12559028 & All & 0.49 & SJC & 905 & 2.182 & 0.5349 & $4.9 \mathrm{E}-05$ & 0.050 \\
& & & rs5943618 & All & 0.44 & SJC & 905 & 2.27 & 0.5424 & $3.1 \mathrm{E}-05$ & 0.046 \\
& & & rs4829239 & All & 0.38 & SJC & 905 & 2.321 & 0.5592 & $3.6 \mathrm{E}-05$ & 0.046 \\
& & & rs5927671 & All & 0.45 & ESR & 897 & 0.1181 & 0.02853 & $3.8 \mathrm{E}-05$ & 0.046 \\
\hline
\end{tabular}

All results for serum markers are shown. For RNA, the probe sets within each gene were strongly correlated. One probe set each in IL-6R and IL-6ST is shown. For DNA, only associations with raw $p<0.01$ (tier 1) and FDR ps $<0.05$ (tiers 2 and 3 ) were shown. FDR was calculated within the population (white or all) and within the tier.

$\beta$, regression coefficient, representing the difference in the end point corresponding to SD in marker value; Chr, chromosome; CRP, C reactive protein; DAS28, Disease Activity Score using 28 joints; DMARD, disease-modifying antirheumatic drug; ESR, erythrocyte sedimentation rate; HAQ, Health Assessment Questionnaire; IL-6ST, IL-6 signal transducer; IR, inadequate responder; MAF, minor allele frequency; MTX, methotrexate; SJC, swollen joint count; TCZ, tocilizumab; TJC, tender joint count.

pooled DMARD-IR population and aTNF-IR population. The distribution of baseline IL-6 was similar in the rheumatoid factor-positive and rheumatoid factornegative subpopulations $(45.2 \pm 60.5$ and $41.5 \pm 60.6$ (mean $\pm \mathrm{SD})$, respectively).
Associations with baseline disease activity

Higher baseline serum IL-6 levels were significantly associated with higher baseline DAS28, ESR, CRP and HAQ in all three populations analysed, with $\mathrm{p}<0.0001$ in most cases (table 3). The strongest association was observed 


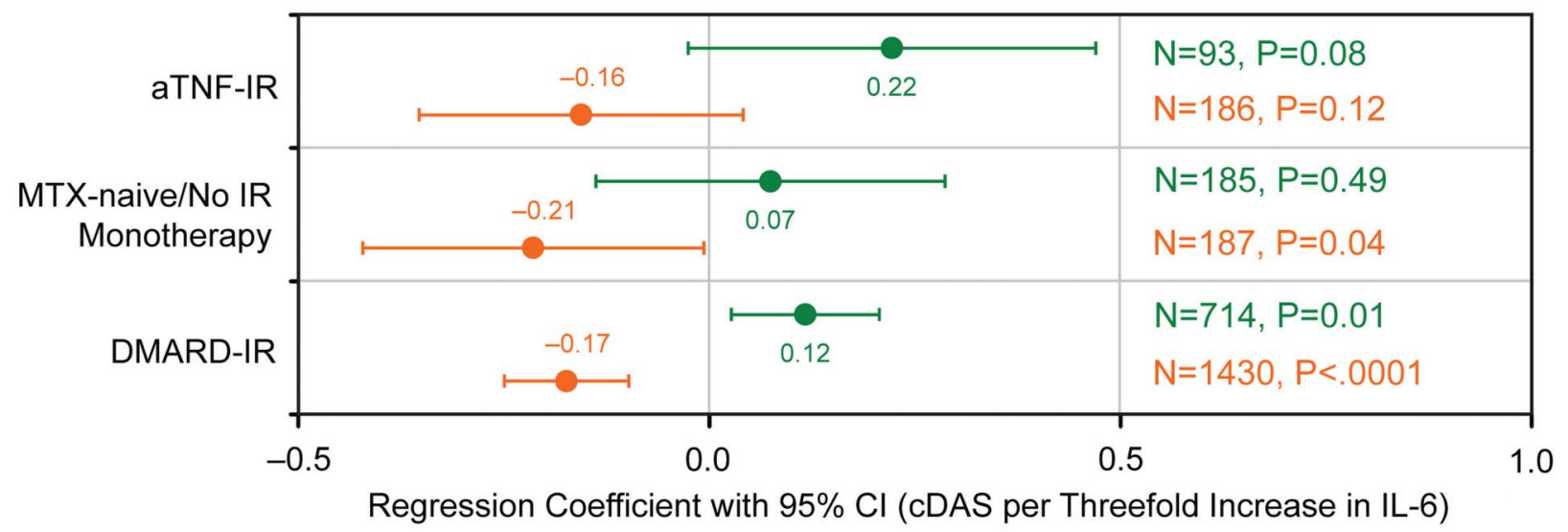

Figure 1 Serum interleukin 6 (IL-6) concentration association with response to treatment. Forest plots show the effect and 95\% $\mathrm{Cl}$ for the association of IL-6 with cDAS28 at 16 weeks across treatment lines. cDAS28, change in DAS28 from baseline at week 16; Cl; DMARD, disease-modifying antirheumatic drug; IR, inadequate responder; MTX, methotrexate; SD; TCZ, tocilizumab; TNF, tumour necrosis factor; 0 , MTX/DMARD; 0 , TCZ+MTX/DMARD.

between CRP and IL-6, for a correlation coefficient of $0.29-0.36$ in three populations (see online supplementary figure S1B). IL-6 was not strongly associated with SJC28 or TJC28. Baseline sIL-6R was not strongly associated with any baseline disease activity measures.

Among the baseline RNA transcripts, an association was observed between the IL-6R transcript and CRP and ESR at baseline (data not shown). No association was found with the other baseline disease activity measures. Association with CRP and ESR was also observed, to a lesser degree, for an IL-6ST transcript.

After correction for multiple testing, no significant associations were found for any of the $I L-6$ or $I L-6 R$ polymorphisms genotyped with baseline disease activity. Of the 1237 polymorphisms representing 22 genes in the IL-6 canonical pathway, 67 additional genes in the IL-6 network, six baseline disease activity end points and two patient populations (all-patient, white), six associations were found to have borderline significance after adjusting for multiple testing, with an FDR of approximately 5\%. There were SNPs in TOLLIP and RUNX1 versus ESR and SNPs in ILIRAPL1 versus SJC and ESR. Such borderline significance meant that the association should be treated as a weak hypothesis.

\section{Associations with clinical response to tocilizumab}

Higher baseline serum IL-6 concentrations were significantly associated with better clinical response to tocilizumab but not to placebo, as measured by cDAS28 in the pooled DMARD-IR population $(\mathrm{p}<0.0001$; see online supplementary table S2, figure 1). The same effect was observed in the MTX-naive population $(p=0.04)$. In the aTNF-IR population, the association was not significant, partially because of the smaller sample size. In the DMARD-IR population, a threefold difference in baseline IL- 6 concentration, equivalent to $1 \mathrm{SD}$ in the baseline $\log ($ IL-6) distribution, corresponded to a small difference of 0.17 units in cDAS28 score at week 16 . The association between serum IL-6 level and achievement of DAS28 remission (DAS28<2.6) was analysed using receiver operating characteristic analysis in the pooled DMARD-IR population treated with tocilizumab $(\mathrm{n}=1547)$. The area under the curve was only 0.59 , suggesting that baseline serum IL-6 levels provided very little discrimination between those achieving and those not achieving DAS28 remission. In the placebo group, the direction of association was opposite that of tocilizumab-treated patients, indicating that baseline serum IL-6 level (with no longitudinal data on change in level) is not a prognostic factor for clinical response in RA in these cohorts.

Baseline sIL-6R concentration in serum had no effect on treatment response in either the tocilizumab or the placebo group (see online supplementary table S2). IL-6R whole blood mRNA (in RADIATE) was not associated with clinical response to tocilizumab or placebo treatment (see online supplementary table S2). No $I L-6$ or $I L-6 R$ polymorphisms were associated with cDAS28 after correction for multiple testing. Although rs2069840 and rs12700386 had a raw $\mathrm{p}<0.05$ (table 4), the estimated effect size was small. Each copy of the minor allele for rs2069840 corresponded to only a 0.3-unit difference in cDAS28. Of the SNPs in the canonical and the network or signalling pathway, rs973767 (an intronic SNP in PTPN2) had the lowest $\mathrm{p}$ value $\left(5.7 \times 10^{-5}\right)$ but was not significant after correction for multiple testing (FDR $=0.057$; table 4 ). In the rheumatoid factor-positive subpopulation, the effect of baseline serum IL-6 on clinical response was very similar to that in the overall population (see online supplementary figure S3).

\section{DISCUSSION}

We have investigated the associations among a number of DNA, RNA and protein biomarkers directly related to IL-6 signalling (table 2) with baseline disease activity 
Table 4 Association of baseline markers with treatment response measured by change in DAS28 from baseline at week 16

\begin{tabular}{|c|c|c|c|c|c|c|c|c|c|c|c|}
\hline \multicolumn{12}{|c|}{ All association results for primary DNA markers } \\
\hline \multirow[b]{2}{*}{ Gene } & \multirow[b]{2}{*}{ SNP } & \multicolumn{5}{|c|}{ All DMARD-IR, TCZ } & \multicolumn{5}{|c|}{ White DMARD-IR, TCZ } \\
\hline & & MAF & $\mathbf{n}$ & $\boldsymbol{\beta}$ & SE & Raw P & MAF & $\mathbf{n}$ & $\boldsymbol{\beta}$ & SE & Raw P \\
\hline \multirow[t]{10}{*}{$I L-6$} & rs12700386 & 0.19 & 583 & 0.22 & 0.11 & 0.049 & 0.18 & 468 & 0.16 & 0.13 & 0.20 \\
\hline & rs2069833 & 0.35 & 584 & -0.08 & 0.08 & 0.34 & 0.42 & 469 & -0.13 & 0.09 & 0.18 \\
\hline & rs2069837 & 0.08 & 586 & -0.11 & 0.15 & 0.44 & 0.08 & 471 & -0.05 & 0.16 & 0.75 \\
\hline & rs2066992 & 0.12 & 586 & -0.09 & 0.12 & 0.49 & 0.06 & 471 & -0.11 & 0.21 & 0.61 \\
\hline & rs2069840 & 0.32 & 584 & 0.24 & 0.09 & 0.007 & 0.33 & 469 & 0.30 & 0.10 & 0.0026 \\
\hline & rs1554606 & 0.4 & 586 & -0.10 & 0.08 & 0.23 & 0.45 & 471 & -0.15 & 0.09 & 0.12 \\
\hline & rs2069845 & 0.4 & 583 & -0.10 & 0.08 & 0.23 & 0.45 & 468 & -0.15 & 0.09 & 0.12 \\
\hline & rs2069861 & 0.07 & 584 & -0.10 & 0.15 & 0.53 & 0.08 & 469 & -0.11 & 0.16 & 0.50 \\
\hline & rs10242595 & 0.36 & 586 & -0.02 & 0.08 & 0.86 & 0.32 & 471 & -0.02 & 0.10 & 0.80 \\
\hline & rs11766273 & 0.07 & 586 & 0.10 & 0.16 & 0.53 & 0.08 & 471 & 0.05 & 0.17 & 0.75 \\
\hline \multirow[t]{18}{*}{$I L-6 R$} & rs1386821 & 0.15 & 586 & 0.02 & 0.12 & 0.87 & 0.17 & 471 & 0.06 & 0.13 & 0.67 \\
\hline & rs4075015 & 0.39 & 584 & -0.06 & 0.09 & 0.50 & 0.41 & 469 & -0.07 & 0.10 & 0.46 \\
\hline & rs6684439 & 0.41 & 586 & 0.03 & 0.08 & 0.76 & 0.39 & 471 & 0.06 & 0.10 & 0.55 \\
\hline & rs4845618 & 0.43 & 582 & 0.03 & 0.08 & 0.74 & 0.44 & 467 & 0.00 & 0.09 & 0.99 \\
\hline & rs8192282 & 0.15 & 581 & -0.09 & 0.12 & 0.46 & 0.16 & 468 & -0.08 & 0.13 & 0.53 \\
\hline & rs7549250 & 0.43 & 575 & 0.07 & 0.08 & 0.43 & 0.44 & 462 & 0.02 & 0.09 & 0.81 \\
\hline & rs4553185 & 0.43 & 586 & 0.04 & 0.08 & 0.60 & 0.44 & 471 & 0.01 & 0.09 & 0.90 \\
\hline & rs4845623 & 0.44 & 586 & -0.01 & 0.08 & 0.92 & 0.41 & 471 & 0.01 & 0.10 & 0.89 \\
\hline & rs4537545 & 0.44 & 586 & -0.04 & 0.08 & 0.60 & 0.41 & 471 & -0.01 & 0.10 & 0.93 \\
\hline & rs4129267 & 0.41 & 586 & -0.02 & 0.08 & 0.77 & 0.4 & 471 & 0.02 & 0.10 & 0.87 \\
\hline & rs8192284 & 0.41 & 582 & -0.03 & 0.08 & 0.76 & 0.4 & 467 & 0.02 & 0.10 & 0.87 \\
\hline & rs11265618 & 0.17 & 586 & -0.05 & 0.11 & 0.64 & 0.17 & 471 & -0.04 & 0.13 & 0.73 \\
\hline & rs4329505 & 0.16 & 582 & -0.03 & 0.11 & 0.81 & 0.16 & 467 & -0.02 & 0.13 & 0.87 \\
\hline & rs4240872 & 0.24 & 586 & 0.03 & 0.10 & 0.79 & 0.23 & 471 & -0.01 & 0.11 & 0.93 \\
\hline & rs4509570 & 0.24 & 580 & 0.02 & 0.10 & 0.85 & 0.23 & 466 & -0.02 & 0.11 & 0.83 \\
\hline & rs2229238 & 0.19 & 584 & -0.03 & 0.11 & 0.75 & 0.19 & 469 & 0.00 & 0.12 & 0.98 \\
\hline & rs7514452 & 0.19 & 586 & -0.03 & 0.11 & 0.77 & 0.19 & 471 & 0.00 & 0.12 & 0.98 \\
\hline & rs7526293 & 0.2 & 584 & -0.04 & 0.10 & 0.71 & 0.2 & 469 & -0.03 & 0.12 & 0.79 \\
\hline
\end{tabular}

DNA markers analysed in the pooled DMARD-IR population with an FDR ${ }^{*}$ of $<0.1$

\begin{tabular}{llllllllll} 
Tiers & Gene & SNP & Population & MAF & $\mathbf{n}$ & $\boldsymbol{\beta}$ & SE & RAW P & FDR P \\
\hline 1 & IL-6 & rs2069840 & White & 0.33 & 469 & 0.30 & 0.10 & 0.0026 & 0.073 \\
3 & PTPN2 & rs973767 & All white & 0.14 & 586 & 0.48 & 0.12 & $7.2 \mathrm{E}-05$ & 0.072 \\
& & & & 0.16 & 471 & 0.53 & 0.13 & $5.7 \mathrm{E}-05$ & 0.057 \\
\hline
\end{tabular}

Baseline DAS28 and TCZ dose were included in the model as covariates.

${ }^{*}$ FDR was calculated within the white or all population and within the tier.

$\beta$, regression coefficient, representing the difference in DAS28 corresponding to each SD in marker value; DAS28, Disease Activity Score using 28 joints; DMARD, disease-modifying antirheumatic drug; FDR, false discovery rate; IL-6, interleukin-6; IL-6R, IL-6 receptor; IR,

inadequate responder; MAF, minor allele frequency; SNP, single nucleotide polymorphism; TCZ, tocilizumab.

and treatment benefit from tocilizumab (table 1). We demonstrate the modest association between serum IL-6 levels and clinical disease activity as measured by DAS28 and HAQ. Although serum IL-6 levels have previously been shown to be elevated in patients with RA compared with controls, ${ }^{92}$ this is the first time a significant correlation between IL-6 level and disease activity has been demonstrated in a large population. IL-6 level accounted for only a small component of observed variance in disease activity (see online supplementary table S2, figure 1). Of the four DAS28 components, serum IL-6 level was most strongly associated with ESR/CRP. This is not surprising given the pivotal role of IL-6 (and the pronounced inhibitory effect of tocilizumab) on the acute-phase response. ${ }^{25}$ Interestingly, HAQ, a measure of patient-reported disability independent of inflammation, also had a significant association with serum IL-6 level.

Littman $^{26}$ proposes that IL-6/tocilizumab may represent 'low-hanging fruit' in terms of personalised healthcare in RA, but our analysis suggested otherwise. Higher baseline DAS28 was associated with higher change in DAS28 in response to therapy (see online supplementary figure S1C). When change in DAS28 was corrected for baseline DAS28, only a weak association with serum IL-6 was observed. Although IL-6 has been shown to decrease with effective treatments, such as modified-release prednisone, ${ }^{27}$ in RA, we saw no evidence that a single baseline 'snapshot' serum IL-6 level was a general prognostic factor for clinical response in 
RA (ie, predicted subsequent treatment response or disease course).

Serum IL-6 exhibits a pronounced circadian rhythm. ${ }^{28}$ This source of variability was minimised, but not eliminated, by collecting the samples in as narrow a time window as achievable in large clinical studies. It is possible that discordance in IL-6 signalling activity between blood and synovial tissue explains the lack of association with blood samples in our study. However, this cannot explain the negative genetic data. The lack of genetic associations between polymorphisms in $I L-6 R$ (including those determining sIL-6R levels) and baseline disease activity $^{1129}$ shows that sIL-6R levels, although highly variable, are not appreciably different between patients who have RA and those who are healthy. ${ }^{30} 31$ The effect of a genetic marker can be affected by a patient's genetic background. An ethnically homogeneous population is ideal for genetic analysis. In this cohort, $79 \%$ of the patients were of European ancestry (White). All genetic analyses were performed in the overall population for maximum sample size and in the White subpopulation for genetic homogeneity. The White subpopulation was sufficiently large; therefore, the lack of a strong genetic effect is compelling.

Formal multiplicity adjustment was not carried out in the analysis of serum and RNA markers. However, informal assessment was used to help interpret the results of the statistical analysis. Multiplicity of the statistical testing was indicated by multiple markers and multiple end points; the numbers of markers in each sample type are shown in table 2. Six baseline disease characteristics and one clinical response end point were used. In addition, genetic analyses were performed in the White and the overall populations. Overall, $\geq 10$ tests were carried out for each serum and RNA marker; results with $\mathrm{p}<0.0001$ would have been significant had adjustment for multiplicity been performed, and results with unadjusted $\mathrm{p}>0.01$ can be regarded as likely false positives.

Power assessment was not performed. Empirically, given the large sample size for DNA and serum samples, the power to detect a clinically relevant effect at $\alpha=0.05$ was very high. Therefore, $\mathrm{p}>0.05$ can be interpreted as evidence of no effect. Analyses of RNA markers had low power because of the limited sample size.

Our analyses illustrate that in a disease such as RA, variation in the abundance of a therapeutic target may not be a strong predictor of patient benefit. In particular, the response to tocilizumab was not dependent on IL-6R SNPs, which were found to be associated with asthma and cardiovascular risk. In addition, a genomewide association approach using 1600 patients from five controlled clinical trials did not yield any strong predictor to tocilizumab treatment response. ${ }^{32}$ These data suggest that the major contribution to variability in clinical responsiveness to therapeutics in RA remains unknown, and they illustrate the challenges of identifying predictive biomarkers, even in large, well-conducted studies.
Acknowledgements The authors would like to thank Stefan Palme for providing the IL-6 data measured on the IMPACT platform; Chih Jian Lih and Mickey Williams for providing the gene expression data; Yan Li for help with the gene expression data QC; Teodorica Bugawan for providing the HLA-DRB1 and shared epitope data; Delphine Lagarde for the DNA and RNA sample preparation and the method description; Gregg Silverman, Ann Begovich, Paul Cutler and Friedemann Krause for helpful discussions; and Santo D'Angelo, PhD, for third-party writing assistance.

Contributors JW designed the study, conducted research, conducted the literature search, analysed and interpreted the data and drafted the manuscript. AP designed the study, conducted research, conducted the literature search, collected the data, analysed and interpreted the data, oversaw data collection and drafted the manuscript. RU analysed and interpreted the data and drafted the manuscript. SG designed the study and collected the data. GL analysed and interpreted the data. CR analysed and interpreted the data. RB designed the study, collected and analysed the data. AK analysed and interpreted the data. AH designed the study and collected the data. MM designed the study. $\mathrm{OH}$ interpreted the data and drafted the manuscript.

Funding This study was funded by Roche. Support for third-party writing assistance for this manuscript was provided by F. Hoffmann-La Roche Ltd.

Competing interests All authors are (or were at the time of the study) employees of Roche or Genentech, a member of the Roche group, and four authors (AH, AK, $\mathrm{OH}$ and $\mathrm{RU})$ also own stock in Roche.

\section{Patient consent Obtained.}

Ethics approval The study protocols were approved by relevant institutional review boards or ethics committees, and written informed consent was obtained from each patient. The studies were carried out in full concordance with the principles of the Declaration of Helsinki and with the laws and regulations of the country in which the research was conducted. DNA and RNA samples were collected only from patients who gave separate informed consent.

Provenance and peer review Not commissioned; externally peer reviewed.

Data sharing statement No additional data are available.

Open Access This is an Open Access article distributed in accordance with the Creative Commons Attribution Non Commercial (CC BY-NC 3.0) license, which permits others to distribute, remix, adapt, build upon this work noncommercially, and license their derivative works on different terms, provided the original work is properly cited and the use is non-commercial. See: http:// creativecommons.org/licenses/by-nc/3.0/

\section{REFERENCES}

1. Cronstein BN. Interleukin-6-a key mediator of systemic and local symptoms in rheumatoid arthritis. Bull NYU Hosp Joint Dis 2007;65: S11-15.

2. Wrighting DM, Andrews NC. Interleukin-6 induces hepcidin expression through STAT3. Blood 2006;108:3204-9.

3. Smolen JS, Beaulieu A, Rubbert-Roth A, et al. Effect of interleukin-6 receptor inhibition with tocilizumab in patients with rheumatoid arthritis (OPTION study): a double-blind, placebo-controlled, randomised trial. Lancet 2008;371:987-97.

4. Emery $\mathrm{P}$, Keystone $\mathrm{E}$, Tony HP, et al. IL-6 receptor inhibition with tocilizumab improves treatment outcomes in patients with rheumatoid arthritis refractory to anti-tumour necrosis factor biologicals: results from a 24-week multicentre randomised placebo-controlled trial. Ann Rheum Dis 2008;67:1516-23.

5. Genovese MC, McKay JD, Nasonov EL, et al. Interleukin-6 receptor inhibition with tocilizumab reduces disease activity in rheumatoid arthritis with inadequate response to disease-modifying antirheumatic drugs: the tocilizumab in combination with traditional disease-modifying antirheumatic drug therapy study. Arthritis Rheum 2008;58:2968-80

6. Jones G, Sebba A, Gu J, et al. Comparison of tocilizumab monotherapy versus methotrexate monotherapy in patients with moderate to severe rheumatoid arthritis: the AMBITION study. Ann Rheum Dis 2010;69:88-96.

7. Kremer JM, Blanco R, Brzosko S, et al. Tocilizumab inhibits structural joint damage in rheumatoid arthritis patients with 
inadequate responses to methotrexate: results from the double-blind treatment phase of a randomized placebo-controlled trial of tocilizumab safety and prevention of structural joint damage at one year. Arthritis Rheum 2011;63:609-21.

8. Nishimoto N, Yoshizaki K, Miyasaka N, et al. Treatment of rheumatoid arthritis with humanized anti-interleukin-6 receptor antibody: a multicenter, double-blind, placebo-controlled trial. Arthritis Rheum 2004;50:1761-9.

9. Madhok R, Crilly A, Watson J, et al. Serum interleukin 6 levels in rheumatoid arthritis: correlations with clinical and laboratory indices of disease activity. Ann Rheum Dis 1993;52:232-4.

10. Robak T, Gladalska A, Stepien $\mathrm{H}$, et al. Serum levels of interleukin-6 type cytokines and soluble interleukin-6 receptor in patients with rheumatoid arthritis. Mediators Inflamm 1998;7:347-53.

11. Galicia JC, Tai H, Komatsu Y, et al. Polymorphisms in the IL-6 receptor (IL-6R) gene: strong evidence that serum levels of soluble L-6R are genetically influenced. Genes Immun 2004:5:513-16.

12. Marinou I, Walters $\mathrm{K}$, Winfield $\mathrm{J}$, et al. A gain of function polymorphism in the interleukin 6 receptor influences RA susceptibility. Ann Rheum Dis 2010;69:1191-4.

13. Fishman D, Faulds $G$, Jeffery $R$, et al. The effect of novel polymorphisms in the interleukin-6 (IL-6) gene on IL-6 transcription and plasma IL-6 levels, and an association with systemic-onset juvenile chronic arthritis. J Clin Invest 1998;102:1369-76.

14. Hingorani AD, Casas JP. The interleukin-6 receptor as a target for prevention of coronary heart disease: a mendelian randomisation analysis. Lancet 2012;379:1214-24.

15. Sarwar N, Butterworth AS, Freitag DF, et al. Interleukin-6 receptor pathways in coronary heart disease: a collaborative meta-analysis of 82 studies. Lancet 2012;379:1205-13.

16. Ferreira MA, Matheson MC, Duffy DL, et al. Identification of IL6R and chromosome 11q13.5 as risk loci for asthma. Lancet 2011;378:1006-14.

17. Fraunberger $\mathrm{P}$, Pfeiffer $\mathrm{M}$, Cremer $\mathrm{P}$, et al. Validation of an automated enzyme immunoassay for ilnterleukin- 6 for routine clinical use. Clin Chem Lab Med 1998;36:797-801.

18. Claudon A, Vergnaud P, Valverde C, et al. New automated multiplex assay for bone turnover markers in osteoporosis. Clin Chem 2008:54:1554-63.

19. R\&D Systems Inc. Quantikine $\circledast$ ELISA human IL-6 immunoassay. Minneapolis, MN: R\&D Systems Inc., 2011.
20. R\&D Systems Inc. Quantikine $($ ELISA human IL-6 sR immunoassay. Minneapolis, MN: R\&D Systems Inc., 2009.

21. Irizarry RA, Bolstad BM, Collin F, et al. Summaries of AffymetrixGeneChip probe level data. Nucleic Acids Res 2003;31: e15.

22. Gregersen PK, Silver J, Winchester RJ. The shared epitope hypothesis: an approach to understanding the molecular genetics of susceptibility to rheumatoid arthritis. Arthritis Rheum 1987:30:1205-13

23. Benjamini $\mathrm{Y}$, Hochberg $\mathrm{Y}$. Controlling the false discovery rate: a practical and powerful approach to multiple testing. J R Stat Soc 1995:57:289-300

24. Chung SJ, Kwon YJ, Park MC, et al. The correlation between increased serum concentrations of interleukin- 6 family cytokines and disease activity in rheumatoid arthritis patients. Yonsei Med $\mathrm{J}$ 2011;52:113-20.

25. Heinrich PC, Castell JV, Andus T. Interleukin- 6 and the acute phase response. Biochem J 1990;265:621-36.

26. Littman BH. Tocilizumab and missed personalized medicine opportunities for patients with rheumatoid arthritis? Arthritis Rheum 2009;60:1565-6.

27. Buttgereit $F$, Doering G, Schaeffler A, et al. Efficacy of modified-release versus standard prednisone to reduce duration of morning stiffness of the joints in rheumatoid arthritis (CAPRA-1): a double-blind, randomised controlled trial. Lancet 2008;371:205-14.

28. Kirwan JR, Clarke L, Hunt LP, et al. Effect of novel therapeutic glucocorticoids on circadian rhythms of hormones and cytokines in rheumatoid arthritis. Ann NY Acad Sci 2010;1193:127-33.

29. Rafiq S, Frayling TM, Murray A, et al. A common variant of the interleukin 6 receptor (IL-6r) gene increases IL-6r and IL-6 levels, without other inflammatory effects. Genes Immun 2007;8:552-9.

30. De Benedetti F, Pignatti P, Gerloni V, et al. Differences in synovial fluid cytokine levels between juvenile and adult rheumatoid arthritis. J Rheumatol 1997;24:1403-9.

31. Frieling JT, Sauerwein RW, Wijdenes J, et al. Soluble interleukin 6 receptor in biological fluids from human origin. Cytokine 1994;6:376-81.

32. Wang J, Bansal AT, Martin M, et al. Genome-wide association analysis implicates the involvement of eight loci with response to tocilizumab for the treatment of rheumatoid arthritis. Pharmacogenomics J 2013;13:235-41. 